\title{
The VIRUS Data Reduction Pipeline
}

\author{
Claus A. Goessl ${ }^{a}$, Niv Drory ${ }^{b}$, Helena Relke ${ }^{c}$ \\ Karl Gebhardt ${ }^{b}$, Frank Grupp ${ }^{a}$, Gary Hill ${ }^{b}$, Ulrich Hopp ${ }^{a c}$, Ralf Köhler ${ }^{a}$, \\ and Phillip MacQueen ${ }^{b}$ \\ ${ }^{a}$ Universitäts-Sternwarte München, Scheinerstraße 1, D-81679 München, Germany; \\ ${ }^{b}$ The University of Texas, Department of Astronomy, 1 University Station, C1400, Austin, \\ Texas 78712-0259; \\ ${ }^{c}$ Max-Planck-Institut für extraterrestrische Physik, Giessenbachstraße, 85748 Garching, \\ Germany
}

\begin{abstract}
The Hobby-Eberly Telescope Dark Energy Experiment (HETDEX) will measure baryonic acoustic oscillations, first discovered in the Cosmic Microwave Background (CMB), to constrain the nature of dark energy by performing a blind search for Ly- $\alpha$ emitting galaxies within a $200 \mathrm{deg}^{2}$ field and a redshift bin of $1.8<z<3.7$. This will be achieved by VIRUS, a wide field, low resolution, 145 IFU spectrograph. The data reduction pipeline will have to extract $\approx 35.000$ spectra per exposure $(\approx 5$ million per night, i.e. 500 million in total), perform an astrometric, photometric, and wavelength calibration, and find and classify objects in the spectra fully automatically. We will describe our ideas how to achieve this goal.
\end{abstract}

Keywords: Data reduction pipeline, IFU spectroscopy, dark energy

\section{INTRODUCTION}

HETDEX $^{1}$ is a newly designed survey of the distribution of high redshift galaxies which aims at understanding the evolution history of our universe. Based on observations of supernovae of type SN Ia, it was found that about $70 \%$ of the total energy of the Universe consists of this mysterious dark energy. Beyond its bare existence, little is known about dark energy but concepts have been designed to study its properties. HETDEX will allow to describe precisely the expansion history of the Universe, thereby illuminating the nature of the dark energy.

HETDEX should map the distribution of about one million galaxies in a volume ten times larger than those investigated so far (in the redshift $z$ regime $1.8<z<3.7$ ). Existing telescope-instrument combinations are not able to conduct this massive survey. VIRUS, ${ }^{2}$ a new instrument for the Hobby-Eberly Telescope ${ }^{3,4}$ (HET) is specifically designed for this task. Its optimized performance will allow to carry out the HETDEX survey within about 110 clear nights. The central concept of VIRUS is the industrial replication of 145 copies of a simple integral field spectrograph, a new instrument production concept in astronomy. The replication will allow to build not only an efficient, but also much less expensive instrument compared to classical large prototype-like designs where in most cases, only the prototype is built and operated. In our case, the engineering development cost will be spread over all copies, and additional savings result from the application of industrial manufacturing techniques. An obvious consequence of this approach is its massive parallel data output, and, due to the IFU technique, a challenging series of calibration steps which so far are available only in interactive pipelines for existing single-spectrograph IFU systems. The VIRUS data rate will require a robust automatic pipeline from basic removal of the instrument profile up to the extraction of the final catalog of galaxies. To test the whole concept, a prototype is under construction which will be installed at the McDonald $2.7 \mathrm{~m}$ telescope.

To trace the distribution of matter, galaxies emitting $\mathrm{Ly}-\alpha$ in emission (LAE) are selected. At these high redshifts, a large fraction of all galaxies is still actively forming stars (e.g. Gabasch et al. ${ }^{5}$ ) and therefore, many of them show this line in relatively strong emission. Therefore, they are relatively easy to find and their redshifts are straightforward to extract.

Further author information: (Send correspondence to C.A.G.)

C.A.G.: E-mail: cag@usm.uni-muenchen.de, Telephone: +49 8921805972 


\section{VIRUS DATA OUTPUT - THE CHALLENGE}

\subsection{The Instrument}

The VIRUS instrument will be a set of 145 identical IFU spectrographs, covering a 20 arcmin diameter fieldof-view (FoV) of the new HET corrector with 1/9 fill factor. Every IFU covers $0.2 \operatorname{arcmin}^{2}$ and is built of 246 fibers $\left(1 \operatorname{arcsec}^{2} /\right.$ fiber), so this is 35.670 fibers (i.e. spectra) resulting in about 14 million resolution elements per exposure.

\subsection{Data Flow due to the Observational Setup}

The current observational strategy foresees $6 \times 200$ s exposures per pointing distributed among 6 dither positions to fill the inter-fiber gaps of the IFUs. This results in $20 \mathrm{~GB}$ raw data per hour or (including calibration) about 200 GB raw data per night. Reduction including error propagation makes 800 GB for every intermediate step (conversion to float and additional $\chi^{2}$ images). Based on benchmark tests we estimated that completely reducing the data of one detector and one pointing with one "typical" single CPU computer takes about the same time as taking an exposure. This includes all reduction steps described in Sect. 4. This data rate favors a massive parallel approach for the data reduction.

\subsection{Data Format}

The data format will be FITS $^{6,7}$ with one image extension ${ }^{8}$ for every spectrograph unit. Guide probe images shall be added as additional image extensions. All additional instrumental, telescope and environmental status data will be written to the FITS headers (e.g. WCS $^{9-11}$ ).

\section{VIRUS IMAGE SIMULATION SOFTWARE}

To help in assessing the instrument performance during the design phase and to help develop the VIRUS reduction pipeline, an image simulation package was developed. This software VSIM, simulates CCD images as expected from the VIRUS instrument. The simulation software is built around using the output of the optical modeling and ray-tracing software used in the optical design process (ZEMAX) in order to produce images with characteristics resembling the final instrument as closely as possible.

Internally, VSIM is made up of roughly four parts:

- Sampling of the sky with the IFU head,

- Mapping of the fiber position along the pseudo-slit and wavelength onto the CCD,

- Convolution of the spectra with the output fiber profile and the PSF of the spectrograph and camera, and finally,

- Resampling the output of the fibers onto CCD pixels.

In the following we briefly describe each of these stages.

\subsection{Sampling the Sky with the IFU Head}

VSIM implements the IFU head according to the specs of VIRUS ${ }^{2}: 246$ fibers of $200 \mu \mathrm{m}$ diameter each, arranged as 17 rows of 14 and 15 fibers (alternating) in an hexagonal close pack. Each fiber covers an area of 1 square arcsecond (1.13 arcsec diameter) and the overall fill factor is close to 1/3. Maximum area coverage is achieved with 3 exposures. The exact fiber head geometry can be adjusted in the configuration file, if necessary.

The software simulates either a single exposure or a "dither" of 3 exposures with preset offsets to cover the sky contiguously.

The starting point is a columnar ASCII file with source positions, source magnitudes, source redshifts, and source types. Currently sources can be one of (1) a single Gaussian emission line, (2) a power law continuum, or (3) any SED given by an ASCII file with wavelength and flux columns.

Taking the seeing and the sky intensity as a function of wavelength into account, the software generates a spectrum for each fiber in the IFU head. 


\subsection{The Dispersion and Distortion Map}

Using the ray-tracing output from the optical modeling and design software, we model the dispersion relation and the distortion field of the VIRUS optics as realistically as possible.

We generate a bundle of rays along the pseudo-slit for a number of wavelengths and and map the position of these rays on the CCD plane. Two maps are obtained, $x_{i}\left(s_{i}, \lambda_{i}\right)$, and $y_{i}\left(s_{i}, \lambda_{i}\right)$, that relate the $(x, y)$ coordinates on the CCD to position along the pseudo-slit, $s$, and wavelength $\lambda$. Then, we fit a polynomial of 3 rd order (with cross terms) to these maps to obtain a model of the VIRUS spectrograph and camera optics. This fit is an input to VSIM and fully describes the dispersion and distortion solutions of the instrument as well as the reduction factor of the camera.

\subsection{Convolution with the Fiber Profile and Spectrograph PSF}

Now that we know where in the image plane each fiber and wavelength is imaged, we can proceed to form a monochromatic image at these positions. The final spectrum is just a superposition of these monochromatic images.

To this end we again resort to using ray-tracing to determine the PSF of the spectrograph as a function of position along the pseudo-slit and wavelength. We convert a grid of spot-diagrams to energy distributions, and construct a grid of PSFs as a function of position and wavelength from these.

These spot-diagrams only describe the PSF of the spectrograph for a point-like source. However, the input to the instrument consists of fibers with finite diameters. Therefore, the final grid of PSFs is obtained by convolving the optic's PSFs with the light distribution at the output end of the fibers, which for now we assume to be a circular top-hat of the size of the fiber. Note that this image is altered by the distortion map and its gradient.

The PSF at any arbitrary position along the pseudo-slit and wavelength within this grid is obtained by interpolating the neighboring 4 PSFs in the grid, such that the PSF varies smoothly with position in the final image.

\subsection{Resampling onto the CCD}

The final step in simulating the CCD image is to resample the light distribution in the image plane - obtained by looping over fibers and wavelength in the previous step - onto the pixels of the CCD. Once we know the number of electrons in each pixel, we add Poisson noise and read-out noise to the image. The result is saved to a FITS image (or a cube for simultaneous simulation of multiple dithers). Fig. 1 shows an example of a single exposure simulation where one template for an emission line object and a second one for a galaxy taken from the spectral library of the FORS-Deep field ${ }^{12}$ where added together with realistic flux from the sky.

\section{A CONCEPT FOR DATA REDUCTION - THE CURE FOR VIRUS}

\subsection{Parallelizing tasks}

Since the instrument consists of multiple copies of one basic unit the data reduction will follow the same path: Every "exposure" will be split into single frames, each originating from one of the 145 detectors. Then, all frames will be processed in parallel, each on its own computing node (see Fig.3).

\subsection{Raw CCD Frame Reduction}

The "raw" reduction of the IFU spectra images removes or at least quantifies the detector imprint on the data. This involves dealing with the detector bias, detector bad pixels, pixel sensitivity variations (CCD-Flat), fiber throughput variations (fiber flat), masking of so called cosmic-ray hits (cosmics), and keeping track of the impact of different noise sources (i.e. error propagation).

Bias: The usual strategy to get rid of the detector bias is twofold: By building the average over many bias frames a high $S / N$ bias pattern can be constructed and subtracted which is extremely important when trying to find a weak signal in the detector noise dominated regime (VIRUS will operate at $340<\lambda / \mathrm{nm}<570$ ). The actual bias level as well as the initial error estimate due to the detector read-out noise can be estimated from the overscan of a frame. 


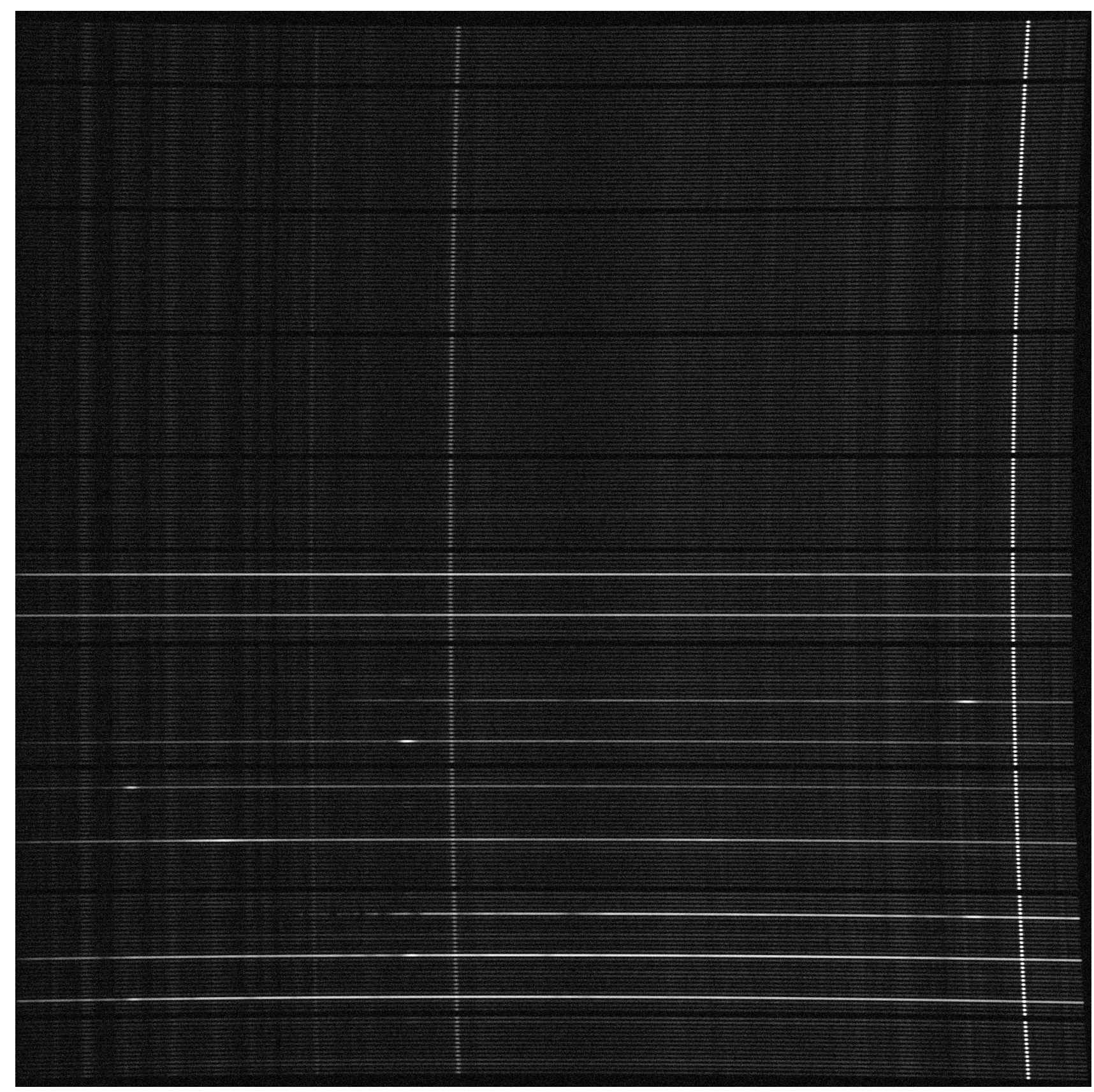

Figure 1. Simulated image of a single IFU/detector unit using realistic night sky emission and template spectra from the FORS Deep Field project for star forming emission line galaxies (ELG) and two LAE of different LA emission line strength. A QSO from the same source was also added. Object spectra visible from bottom to top: The strong-line LAE was placed at redshifts of $z=2.0, z=2.5$, and $z=3.5$; a QSO at a redshift of $z=2.1$; the weak-line LAE was also inserted for $z=2.0, z=2.5$, and $z=3.5$. The ELG was shifted to $z=0.8$ and $z=1.2$. Note that those ELG can be a confusion source for the final LAE object catalog. 

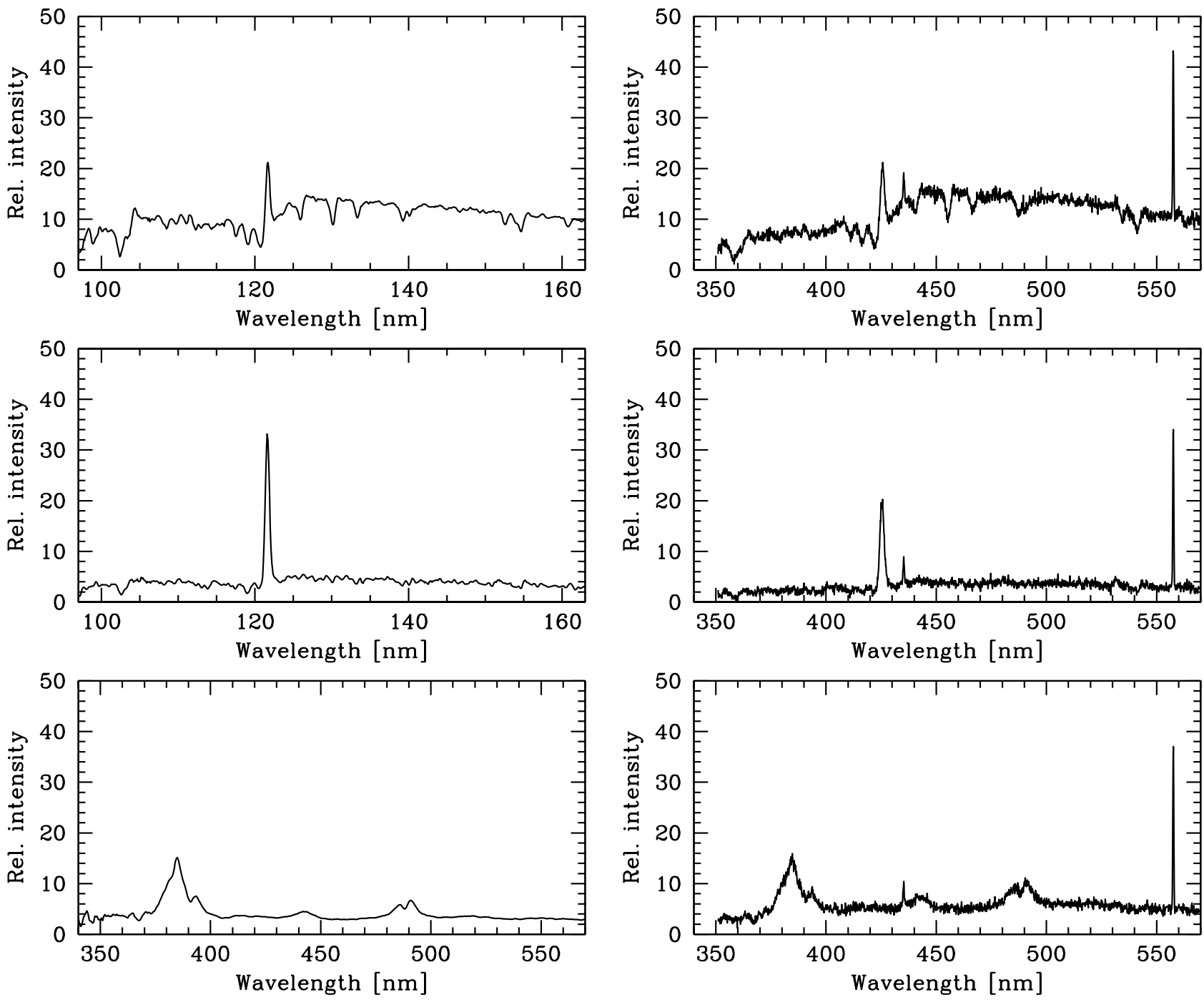

Figure 2. The input (left) and the extracted (right) spectra of Fig. 1. We used an adjusted version of the PMAS/PPAK software package ${ }^{13,14}$ for extraction and wavelength calibration of the simulated spectra. Top: Weak LAE; left is rest frame template, right is simulation for $z=2.5$. Center: Strong LAE; left is rest frame template, right is simulation for $z=2.5$ Bottom: QSO at $z=2.1$. The strong line at $577 \mathrm{~nm}$ is a bright night sky emission line and clearly visible in all the spectra of Fig. 1. 


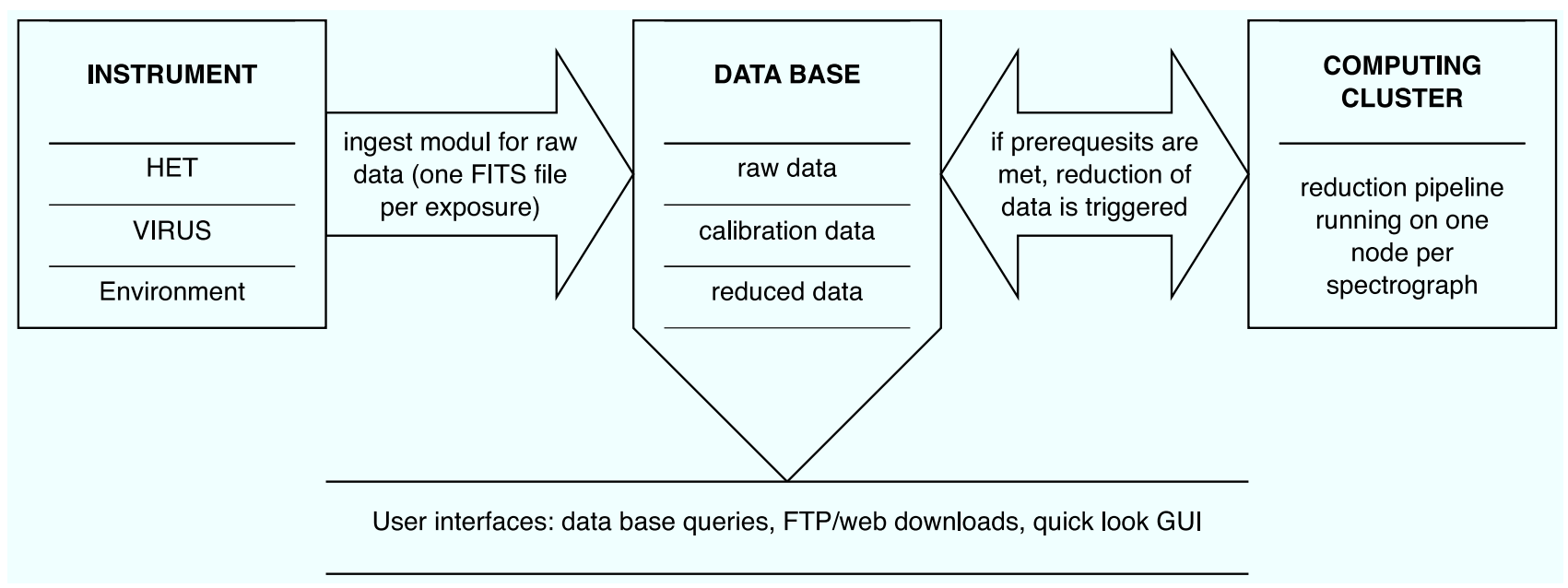

Figure 3. Sketch of the experiment's data flow.

Photon Noise: After bias subtraction the flux in detector units (ADUs) will be translated to photons by multiplying with the gain of the detector. The initial error estimate can now be expanded with the photon noise $\left(\approx \sqrt{n_{\text {photons }}}\right)$ and saved (for easier computation its square) in an FITS image extension.

Bad pixel mask: Saturated pixels will be flagged in the error image according to a saturation level from the FITS header. A bad pixel mask with hot and cold pixels as well as traps will be constructed from darks and flats when testing the detectors and spectrograph units in the lab, stored to the data base and applied to the spectra images. Dark tests will be repeated frequently during operation which can yield updates of the bad pixel mask.

Flatfielding: Small scale sensitivity variations of the detector will be calibrated using high $S / N$ flatfields from lab measurements. The larger scale sensitivity variations due to telescope, spectrograph, camera, and detector coating will be "flatfielded" using continuum lamp and twilight exposures.

Cosmics rejection: The spectrograph design foresees a good sampling of the spectra. Therefore, the steep rise of a cosmics hit can be easily distinguished from a real emission line by a rejection algorithm working on single frames, e.g. by applying the algorithm of Gössl \& Riffeser. ${ }^{15,16}$

Straylight illumination correction: The fibers of each IFU are forming a pseudo-slit with 10 banks of 14, 15, and 29 fibers (see Fig. 1) and about one fiber wide intrabanks gaps. The big intrabank gaps, projected by the spectrograph onto the detector, can serve as a tracer for straylight illumination. A low order polynomial fit to the flux in the gaps will be subtracted from the spectra frames to correct for straylight illumination.

Error propagation: The error image extension for every spectra image holds either the $\chi^{2}$ for detector readout noise plus the photon noise or an integer negative value indicating the cause for being flagged as a bad pixel. For the $\chi^{2}$ case Gaussian propagation is applied throughout "pixel"-based reduction (i.e. subtraction of bias, division by flatfields, exemplary implementation ${ }^{15,16}$ ).

After raw reduction the data will look as close as possible like those of an "ideal" or the simulated instrument (see Fig. 1). 
FITS file: one exposure, holds 145 IFU spectra images + guide probe images in image extensions

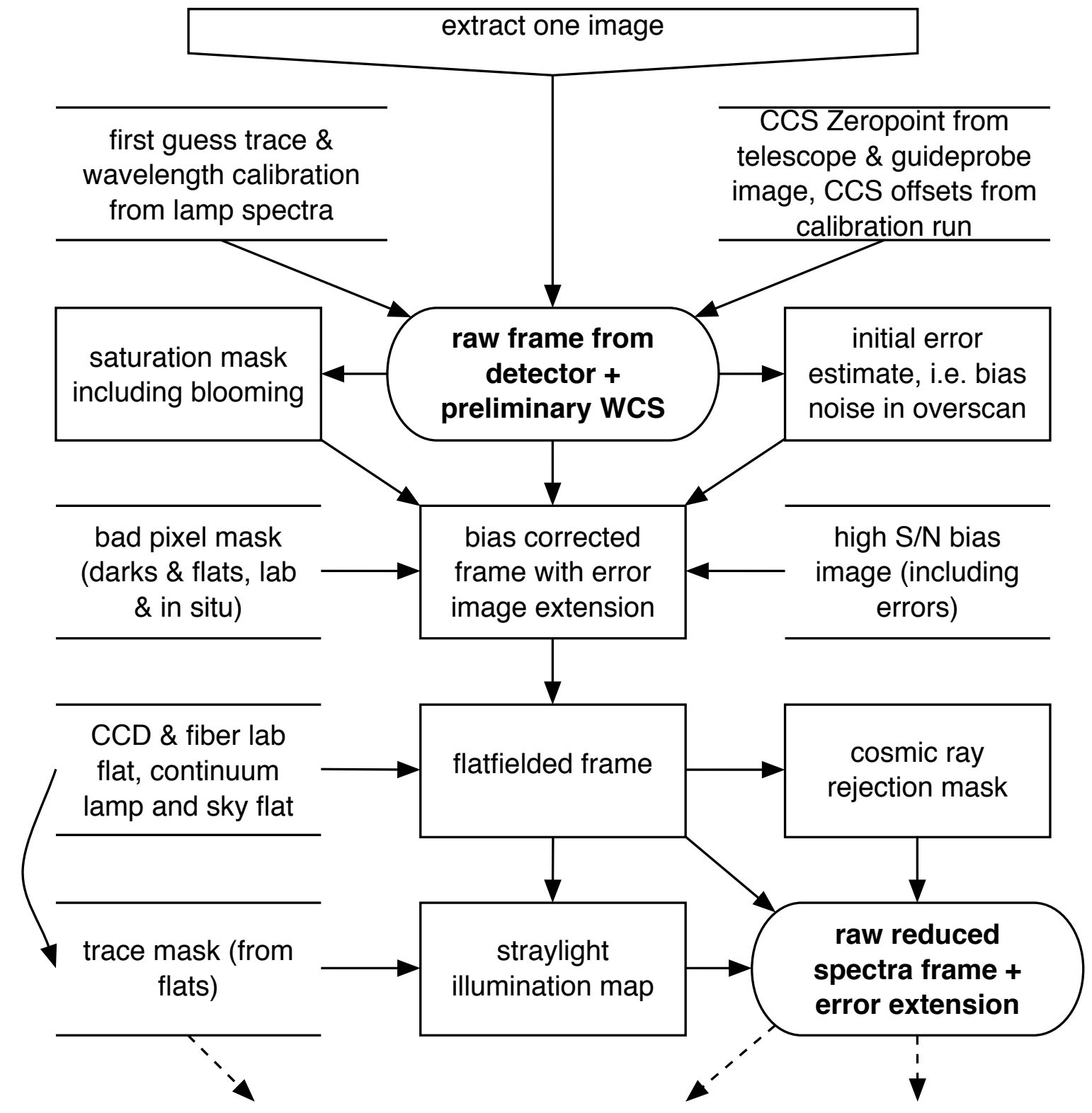

Figure 4. Sketch of the raw reduction pipeline: open boxes involve external input from the data base, round boxes will be results added to the data base. 


\subsection{World Coordinate System}

A first guess WCS will be constructed from telescope data in combination with calibration observations: The HET pointing data and, in case of an unambiguous identification, guide probe stars will give a reference point for the celestial coordinate system (CCS: $R A, D e c$ ). Calibration observations of open clusters add to the fiber position mapping from lab measurements to establish a relative spatial grid. A preliminary wavelength calibration and optical distortion parameters from lab and calibration observations complete the initial WCS FITS header data. The WCS data will be refined later on (see following sections).

\subsection{Tracing}

Due to the fixed elevation of the HET ${ }^{3,4}$ and the fixed mounting of the VIRUS spectrographs we expect very stable instrument performance. Therefore tracing the spectra on the images should be simple using either continuum lamp or sky flats. Tracing information will be stored in the FITS Extension Headers as an addition to the WCS.

\subsection{Wavelength Calibration}

Again we expect very stable instrument performance, so it should be sufficient to obtain only few calibration exposures with emission line lamps and check the calibration with sky emission lines.

\subsection{Skyline subtraction}

Following the recipe, described below in Sect. 4.9 for objects of scientific interest, we will create an accurate map of the night sky lines on the spectra frames which will be subtracted. (Instead of convolution with a stellar PSF a clipped average of all fibers of one IFU will yield the input for the simulator fit run.) The error propagation takes care of not loosing track on the high noise sky line regions.

\subsection{Astrometric Calibration}

The refined astrometric solution will be made by mimicking SDSS broadband images ${ }^{17,18}$ and comparing with the astrometry ${ }^{19}$ of the SDSS catalog. ${ }^{20}$ We estimate to have $\approx 35$ SDSS stars sampled by the IFUs FoV in every exposure at the foreseen HETDEX survey fields.

\subsection{Photometric Calibration}

The overall vignetting of the system will be once observed by measuring the flux in a dense stellar field like e.g. an open cluster with many stars of known magnitudes. During the survey observations, the throughput of the total system (including the atmosphere) should be known on the $10 \%$ level for every exposure. This will be achieved by identifying the stars already mapped by the Sloan Digital Sky Survey. ${ }^{17,18}$

\subsection{Emission Line Detection}

We expect to find emission line candidates following this recipe: The spectra will be convolved with a spectral line model and rebinned to a uniform wavelength grid (correlated noise within size of the convolution kernel). Spatial convolution of a complete set of dithers with a stellar PSF yields a "spaxel" based probability function in terms of $S / N$ for an emission line in the $\lambda-R A-D e c$ space. The noise of close spaxels is correlated within the limits of the convolution kernels, but nevertheless a meaningful indicator. Since the calculation of this probability function has no iterative steps and only requires multiplication and addition it will be very fast. Candidates above a detection threshold now can be back projected via the simulator onto the reduced spectra frame. The final $\lambda, R A, D e c$, and flux will be fitted to give a least- $\chi^{2}$ solution for the comparison of the simulated frame with the reduced science frame. In this way it is even possible to quantify the error budget of every parameter ( $\lambda$, position and flux).

\subsection{Data Products}

The raw data, intermediate reduction results which are CPU-time expensive to create, and the final results will be stored to a data base (see Fig. 4 and 5). These are the "image data", i.e. raw data, reduced spectra frames, WCS-refined calibrated spectra frames, quasi-SDSS images, E3D ${ }^{21}$ compliant spectra, as well as result tables, i.e. preliminary and final catalogs of emission line objects $(R A, D e c, z$, flux, error and significance description). 


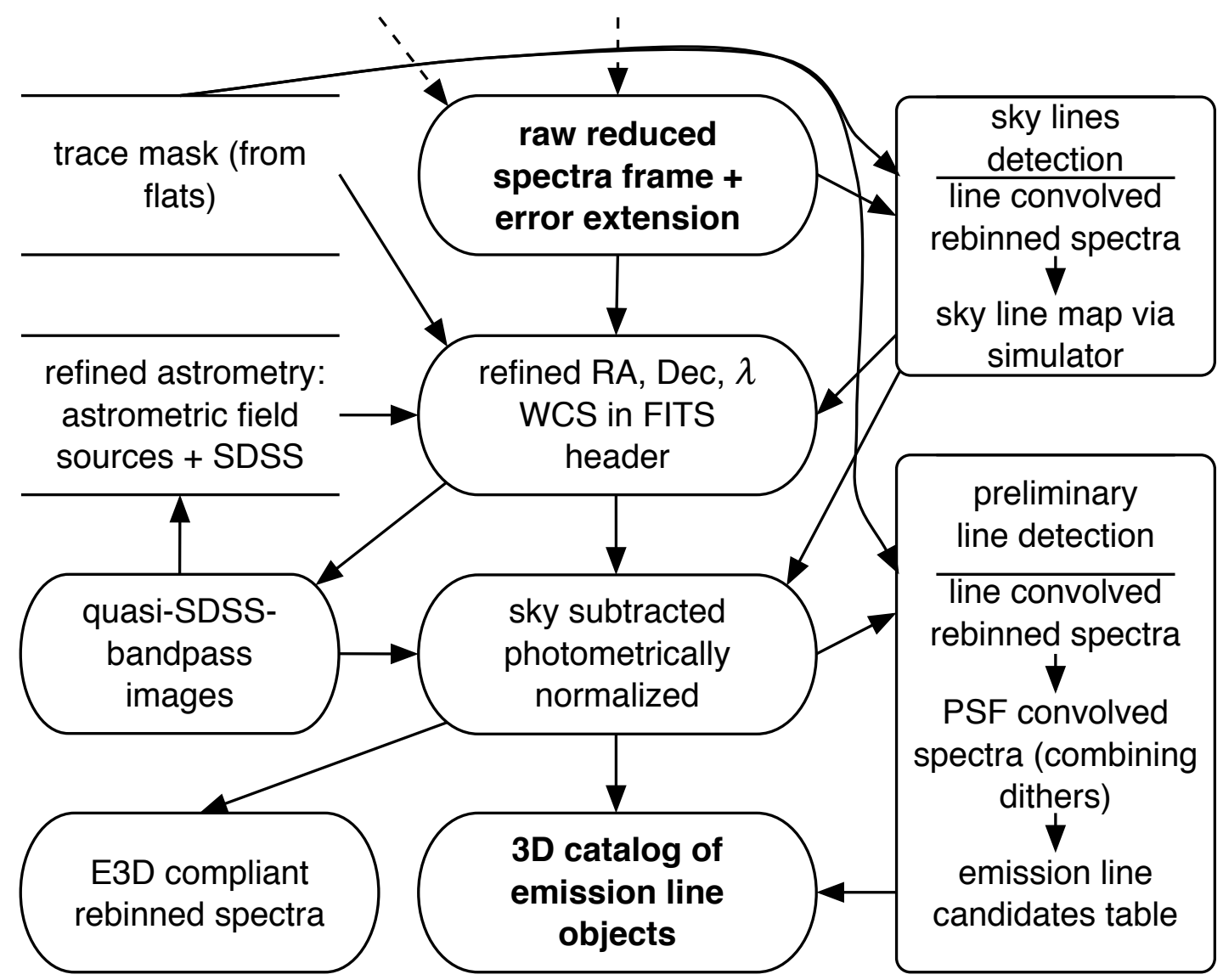

Figure 5. Sketch of the line detection pipeline: open boxes involve external input from the data base, round boxes will be results added to the data base.

\section{CONTROLLING \& USER INTERFACES}

All access to the data will be managed through data base queries. The result of the queries are simple data base fields which can be also links to tables or to spectra images. Reductions will be triggered by specific queries and by watch dog programs, which will check for the necessary prerequisites. The reduction procedures again ingest their results to the data base. Regular calibration runs will have their own special reduction scheme. One time calibration data will be ingested manually.

Example: Quicklook of the data can be achieved by a query for the last exposure transformed to Euro3D format. The resulting link will give a compliant file which can be opened with "your favorite Euro3D spectra viewer".

\section{PROJECT STATUS}

Existing publicly available software packages for reduction of 3D spectra have been evaluated. PMAS-PPAK observations, VIMOS IFU archive data, and VIRUS simulator data have been tested with several existing packages: The PMAS P3D package, the ESO VIMOS pipeline VIPGI, and the more general packages R3D and DoHydra. So far none qualifies for full automatic reduction. We are currently testing dither/stacking strategies and line detection algorithms on real and simulated data sets. The VIRUS prototype (to be deployed in July on the McDonald $2.7 \mathrm{~m}$ telescope) will allow the fine tuning of the software development while its data will be reduced with an interactive pipeline based on the PMAS/PPAK software package. ${ }^{13,14}$ 


\section{ACKNOWLEDGMENTS}

Our thanks are due to M. Roth, A. Kelz and P. Böhm, AIP, for discussion and software support as well as a collaborative effort in hardware development for the VIRUS IFU. We further acknowledge discussions with P. Schuecker, MPE and E. Komatsu, Austin. We like to thank BMBF/DESY (05AV5WMB/6) for their financial support.

\section{REFERENCES}

1. G. Hill, "Hobby-Eberly Telescope Dark Energy Experiment (HETDEX)," in Proceedings of "Probing the Dark Universe with Subaru and Gemini". November 6-9, 2005 AGENDA Waikoloa, Hawaii. http://www.noao.edu/meetings/subaru, p.22.1, Nov. 2005.

2. G. J. Hill, P. J. MacQueen, C. Tejada, F. J. Cobos, P. Palunas, K. Gebhardt, and N. Drory, "VIRUS: a massively replicated IFU spectrograph for HET," in Ground-based Instrumentation for Astronomy. Edited by Alan F. M. Moorwood and Iye Masanori. Proceedings of the SPIE, Volume 5492, pp. 251-261 (2004)., A. F. M. Moorwood and M. Iye, eds., pp. 251-261, Sept. 2004.

3. L. W. Ramsey, M. T. Adams, T. G. Barnes, J. A. Booth, M. E. Cornell, J. R. Fowler, N. I. Gaffney, J. W. Glaspey, J. M. Good, G. J. Hill, P. W. Kelton, V. L. Krabbendam, L. Long, P. J. MacQueen, F. B. Ray, R. L. Ricklefs, J. Sage, T. A. Sebring, W. J. Spiesman, and M. Steiner, "Early performance and present status of the Hobby-Eberly Telescope," in Proc. SPIE Vol. 3352, p. 34-42, Advanced Technology Optical/IR Telescopes VI, Larry M. Stepp; Ed., L. M. Stepp, ed., pp. 34-42, Aug. 1998.

4. J. A. Booth, M. J. Wolf, J. R. Fowler, M. T. Adams, J. M. Good, P. W. Kelton, E. S. Barker, P. Palunas, F. N. Bash, L. W. Ramsey, G. J. Hill, P. J. MacQueen, M. E. Cornell, and E. L. Robinson, "The HobbyEberly Telescope Completion Project," in Large Ground-based Telescopes. Edited by Oschmann, Jacobus M.; Stepp, Larry M. Proceedings of the SPIE, Volume 4837, pp. 919-933 (2003)., J. M. Oschmann and L. M. Stepp, eds., pp. 919-933, Feb. 2003.

5. A. Gabasch, U. Hopp, G. Feulner, R. Bender, S. Seitz, R. P. Saglia, J. Snigula, N. Drory, I. Appenzeller, J. Heidt, D. Mehlert, S. Noll, A. Böhm, K. Jäger, and B. Ziegler, "The evolution of the luminosity functions in the FORS deep field from low to high redshift. II. The red bands," A\&A 448, pp. 101-121, Mar. 2006.

6. D. C. Wells, E. W. Greisen, and R. H. Harten, "FITS - a Flexible Image Transport System," A\&AS 44, pp. 363-+, June 1981.

7. R. J. Hanisch, A. Farris, E. W. Greisen, W. D. Pence, B. M. Schlesinger, P. J. Teuben, R. W. Thompson, and A. Warnock, "Definition of the Flexible Image Transport System (FITS)," A\&A 376, pp. 359-380, Sept. 2001.

8. J. D. Ponz, R. W. Thompson, and J. R. Munoz, "The FITS image extension," A\&AS 105, pp. 53-55, May 1994.

9. E. W. Greisen and M. R. Calabretta, "Representations of world coordinates in FITS," A\&A 395, pp. 10611075, Dec. 2002.

10. M. R. Calabretta and E. W. Greisen, "Representations of celestial coordinates in FITS," A\&A 395, pp. 10771122, Dec. 2002.

11. E. W. Greisen, M. R. Calabretta, F. G. Valdes, and S. L. Allen, "Representations of spectral coordinates in FITS," A\&A 446, pp. 747-771, Feb. 2006.

12. S. Noll, D. Mehlert, I. Appenzeller, R. Bender, A. Böhm, A. Gabasch, J. Heidt, U. Hopp, K. Jäger, S. Seitz, O. Stahl, C. Tapken, and B. L. Ziegler, "The FORS Deep Field spectroscopic survey," A\&A 418, pp. 885906, May 2004.

13. M. M. Roth, A. Kelz, T. Fechner, T. Hahn, S.-M. Bauer, T. Becker, P. Böhm, L. Christensen, F. Dionies, J. Paschke, E. Popow, D. Wolter, J. Schmoll, U. Laux, and W. Altmann, "PMAS: The Potsdam Multi-Aperture Spectrophotometer. I. Design, Manufacture, and Performance," PASP 117, pp. 620-642, June 2005.

14. A. Kelz, M. A. W. Verheijen, M. M. Roth, S. M. Bauer, T. Becker, J. Paschke, E. Popow, S. F. Sánchez, and U. Laux, "PMAS: The Potsdam Multi-Aperture Spectrophotometer. II. The Wide Integral Field Unit PPak," PASP 118, pp. 129-145, Jan. 2006. 
15. C. A. Gössl and A. Riffeser, "Image reduction pipeline for the detection of variable sources in highly crowded fields," A\&A 381, pp. 1095-1109, Jan. 2002.

16. C. A. Gössl and A. Riffeser, "Image Reduction Pipeline for the Detection of Variable Sources in Highly Crowded Fields," in ASP Conf. Ser. 295: Astronomical Data Analysis Software and Systems XII, pp. 229,+ 2003 .

17. M. Fukugita, T. Ichikawa, J. E. Gunn, M. Doi, K. Shimasaku, and D. P. Schneider, "The Sloan Digital Sky Survey Photometric System," AJ 111, pp. 1748-+, Apr. 1996.

18. Ž. Ivezić, R. H. Lupton, D. Schlegel, B. Boroski, J. Adelman-McCarthy, B. Yanny, S. Kent, C. Stoughton, D. Finkbeiner, N. Padmanabhan, C. M. Rockosi, J. E. Gunn, G. R. Knapp, M. A. Strauss, G. T. Richards, D. Eisenstein, T. Nicinski, S. J. Kleinman, J. Krzesinski, P. R. Newman, S. Snedden, A. R. Thakar, A. Szalay, J. A. Munn, J. A. Smith, D. Tucker, and B. C. Lee, "SDSS data management and photometric quality assessment," Astronomische Nachrichten 325, pp. 583-589, Oct. 2004.

19. J. R. Pier, J. A. Munn, R. B. Hindsley, G. S. Hennessy, S. M. Kent, R. H. Lupton, and Ž. Ivezić, "Astrometric Calibration of the Sloan Digital Sky Survey," AJ 125, pp. 1559-1579, Mar. 2003.

20. K. Abazajian, J. K. Adelman-McCarthy, M. A. Agüeros, S. S. Allam, K. S. J. Anderson, S. F. Anderson, J. Annis, N. A. Bahcall, I. K. Baldry, S. Bastian, A. Berlind, M. Bernardi, M. R. Blanton, J. J. Bochanski, W. N. Boroski, H. J. Brewington, J. W. Briggs, J. Brinkmann, R. J. Brunner, T. Budavári, L. N. Carey, F. J. Castander, A. J. Connolly, K. R. Covey, I. Csabai, J. J. Dalcanton, M. Doi, F. Dong, D. J. Eisenstein, M. L. Evans, X. Fan, D. P. Finkbeiner, S. D. Friedman, J. A. Frieman, M. Fukugita, B. Gillespie, K. Glazebrook, J. Gray, E. K. Grebel, J. E. Gunn, V. K. Gurbani, P. B. Hall, M. Hamabe, D. Harbeck, F. H. Harris, H. C. Harris, M. Harvanek, S. L. Hawley, J. Hayes, T. M. Heckman, J. S. Hendry, G. S. Hennessy, R. B. Hindsley, C. J. Hogan, D. W. Hogg, D. J. Holmgren, J. A. Holtzman, S.-i. Ichikawa, T. Ichikawa, Ž. Ivezić, S. Jester, D. E. Johnston, A. M. Jorgensen, M. Jurić, S. M. Kent, S. J. Kleinman, G. R. Knapp, A. Y. Kniazev, R. G. Kron, J. Krzesinski, D. Q. Lamb, H. Lampeitl, B. C. Lee, H. Lin, D. C. Long, J. Loveday, R. H. Lupton, E. Mannery, B. Margon, D. Martínez-Delgado, T. Matsubara, P. M. McGehee, T. A. McKay, A. Meiksin, B. Ménard, J. A. Munn, T. Nash, E. H. Neilsen, H. J. Newberg, P. R. Newman, R. C. Nichol, T. Nicinski, M. Nieto-Santisteban, A. Nitta, S. Okamura, W. O'Mullane, R. Owen, N. Padmanabhan, G. Pauls, J. Peoples, J. R. Pier, A. C. Pope, D. Pourbaix, T. R. Quinn, M. J. Raddick, G. T. Richards, M. W. Richmond, H.-W. Rix, C. M. Rockosi, D. J. Schlegel, D. P. Schneider, J. Schroeder, R. Scranton, M. Sekiguchi, E. Sheldon, K. Shimasaku, N. M. Silvestri, J. A. Smith, V. Smolčić, S. A. Snedden, A. Stebbins, C. Stoughton, M. A. Strauss, M. SubbaRao, A. S. Szalay, I. Szapudi, P. Szkody, G. P. Szokoly, M. Tegmark, L. Teodoro, A. R. Thakar, C. Tremonti, D. L. Tucker, A. Uomoto, D. E. Vanden Berk, J. Vandenberg, M. S. Vogeley, W. Voges, N. P. Vogt, L. M. Walkowicz, S.-i. Wang, D. H. Weinberg, A. A. West, S. D. M. White, B. C. Wilhite, Y. Xu, B. Yanny, N. Yasuda, C.-W. Yip, D. R. Yocum, D. G. York, I. Zehavi, S. Zibetti, and D. B. Zucker, "The Third Data Release of the Sloan Digital Sky Survey," AJ 129, pp. 1755-1759, Mar. 2005.

21. M. Kissler-Patig, Y. Copin, P. Ferruit, A. Pécontal-Rousset, and M. M. Roth, "The Euro3D data format: A common FITS data format for integral field spectrographs," Astronomische Nachrichten 325, pp. 159-162, Mar. 2004. 